Revista de Psicología Vol. 36 (1), 2018 (ISSN 0254-9247)

\title{
Estilos de pensamiento matemático de estudiantes con talento académico ${ }^{1}$
}

\author{
Pamela Reyes-Santander ${ }^{2}$, David Aceituno ${ }^{3}$ y Pablo Cáceres ${ }^{4}$ \\ Pontificia Universidad Católica de Valparaíso, Chile
}

El presente estudio establece el estilo de pensamiento matemático predominante que utilizan los estudiantes con talento académico en la resolución de problemas matemáticos. Los estilos de pensamiento son preferencias por parte de los sujetos en la forma de expresar las habilidades frente a una tarea matemática, en este caso, visual, formal e integrado. En el marco de un estudio ex post facto retrospectivo de grupo único, se evaluó a un total de 99 estudiantes pertenecientes a un programa académico de apoyo al talento con el cuestionario Estilos de Pensamiento Matemático de Borromeo-Ferri. Los resultados indican que los estudiantes declararon orientarse hacia el estilo de pensamiento integrado, que supone el uso de simbología y representaciones verbales junto con expresiones visuales en la resolución de los ejercicios matemáticos, así como una significativa orientación a abordar los problemas de modo combinado, que supone considerar los problemas como un todo.

Palabras claves: estilos de pensamiento matemático, pensamiento visual, pensamiento analítico, talento académico, resolución de problemas.

\section{Mathematical Thinking Styles of Students with Academic Talent}

This study explores the predominant mathematical thinking style that students with academic talent used in solving mathematical problems. Thinking styles are preferences by subjects in the way of expressing mathematical skills against a task, in this case, visual, formal and integrated. We assessed 99 students from an academic support talent program, in a retrospective ex post facto study with only one group. We administered the questionnaire

1 Esta investigación se ha realizado dentro del proyecto PMI/MECESUP CD UCV-1203, subvencionado por la Pontificia Universidad Católica de Valparaíso, Chile.

2 Doctora en Ciencias. Profesora en la Escuela de Pedagogía de la Pontificia Universidad Católica de Valparaíso. Dirección postal: Av. El bosque 1290, Sausalito, Viña del Mar, Chile. Contacto: reyes.santander.pamela@gmail.com

3 Doctor en Historia y doctor en Didáctica de la Historia y de las Ciencias Sociales. Profesor en el Programa BETA-PUCV de la Pontificia Universidad Católica de Valparaíso. Dirección postal: Brasil 2950, Valparaíso, Chile. Contacto: david.aceituno@pucv.cl

4 Doctor en Metodología de la Investigación en Ciencias del Comportamiento. Profesor en la Escuela de Psicología de Pontificia Universidad Católica de Valparaíso. Dirección postal: Av. El bosque 1290, Sausalito, Viña del Mar, Chile. Contacto: pablo@psicometodos.com 
mathematical thinking styles of Borromeo-Ferri and determined that these students exhibited mostly an integrated style of thinking, which involves the use of symbols and verbal representations with visual expressions in solving mathematical exercises. They also show a strong orientation to address the problems of combined mode, which involves considering them as a whole at a time.

Keywords: mathematical thinking styles, visual thinking, analytical thinking, academic talent, problem solving.

\section{Estilos pensamento matemático dos alunos com talento académico}

Este estudo estabelece o estilo predominante do pensamento matemático usado por os alunos com talento acadêmico na resolução de problemas matemáticos. Os estilos de pensamento são as preferências dos indivíduos sobre a forma para expressar as capacidades em uma tarefa matemática, neste caso, visual, formal e integrada. Como parte de um estudo ex post facto retrospectivo de grupo único, foram avaliados um total de 99 estudantes de um programa de talento acadêmico. Foram aplicados nos alunos o questionário "Estilos de Pensamento Matemático de Borromeo-Ferri” e determinou-se que a maioria dos participantes declararam um estilo de pensamento integrado, que envolve o uso de símbolos e representaçôes verbais com resolução de expressóes visuais de exercícios matemáticos. Eles mostram também uma forte orientaçáo para resolver os problemas de modo combinado, o qual envolve a considerá-los como um todo de uma vez.

Palavras-chave: estilos de pensamento matemático, pensamento visual, pensamento analítico, talento acadêmico, resolução de problemas.

\section{Styles de pensée mathématique des étudiants ayant un talent académique}

La présente étude établit le style de pensée mathématique prédominant utilisé par les étudiants ayant un talent académique dans la résolution de problèmes mathématiques. Les styles de pensée sont des préférences de la part des sujets sous la forme d'exprimer les capacités face à une tâche mathématique, dans ce cas, visuelle, formelle et intégrée. Dans une étude rétrospective sur un seul groupe ex post facto, un total de 99 étudiants appartenant à un programme de soutien aux talents universitaires ont été évalués, à qui le questionnaire Styles de Pensée mathématique de Borromeo-Ferri a été appliqué et déterminé que ce type de sujets déclare principalement un style de pensée intégré, ce qui implique l'utilisation de la symbologie et des représentations verbales ainsi que des expressions visuelles dans la résolution des exercices mathématiques. En outre, ils montrent une forte orientation pour aborder les problèmes de manière combinée, ce qui implique de les considérer dans leur ensemble dans le même temps.

Mots-clés: pensée mathématique, styles de pensée, talent académique, résolution des problèmes. 
Entender cómo aprenden los estudiantes se ha convertido en los últimos años en un foco de estudio muy relevante para la investigación educativa, especialmente porque tiene implicancias en el diseño de ambientes de aprendizajes integrados y a la mejora de las prácticas docentes que incidirán en el desarrollo del potencial de los jóvenes (Bransford, Brown \& Cocking, 2002). En este contexto es donde encontramos estudiantes con necesidades educativas especiales, entre las que se cuentan el talento académico o altas capacidades (Tarazona, 2011; Martínez \& Guirado, 2012).

Para trabajar con los estudiantes con talento, se deben considerar algunas de sus características, como la capacidad para presentar altos niveles de atención y concentración, habilidades para trabajar con ideas abstractas y una mayor capacidad para establecer relaciones lógicas, sintetizar y efectuar generalizaciones (Rankin, 2017; Martínez \& Guirado, 2012; Aretzaga et al., 2013). Para potenciar sus habilidades se debe considerar que estos estudiantes tienen un alto desarrollo de la perspicacia, en tanto son agudos observadores y que esto les permite descubrir con facilidad la idea o aspecto central de algún problema o fenómeno (Aretzaga et al., 2013).

Según Martínez y Guirado (2012), la gestión perceptual cumple un rol relevante en los procesos de transformación ideativa de los individuos con talento, donde la visualización sería una de las herramientas que estos estudiantes utilizan cuando entran en situaciones problemas. Otras características de los individuos con talento es la curiosidad y el interés por investigar diversas ideas u objetos, como también tienen una afinidad por la experimentación y una motivación por lo que hacen y aprenden (Aretzaga et al., 2013; Blumen, 2008; Rankin, 2017).

En particular, los jóvenes con talento matemático presentan sus propias características, en el trabajo de Sriraman (2005) se muestra una síntesis de las investigaciones realizadas sobre talento matemático, en las 
cuales se muestra una serie de características individuales relacionadas con las habilidades de estos estudiantes. Algunas de estas características son retomadas en el trabajo de Reyes y Karg (2009), donde destacan para este trabajo el dominio de campos del conocimiento Matemático la persistencia y perseverancia en actividades de la matemática que le motivan y que son de generación metacognitiva, como también la capacidad de captar y manipular información matemática, características que además coinciden con algunas de las propuestas por Artola y Barraca y Mosteiro (2005).

Según lo anterior, al momento de trabajar o participar como docente en un programa de talento académico, se deberían considerar ciertas condiciones o características de estos estudiantes, pero que característica aún no conocida permitiría potenciar más a los estudiantes con talento matemático. El centrarnos en alguna característica cognitiva, que sea visible en las producciones de los estudiantes con talento, daría indicios para el trabajo en matemática en los programas especializados en talento.

Partiendo de la base que cada individuo es diferente y que cada individuo posee diferentes caminos de pensar o modos de pensar, este trabajo se sitúa en una característica cogitiva: los diferentes caminos de expresar o bien los llamados estilos de pensamiento (1997, 2007). En las propias palabras de Sternberg (1994, p. 36): "A style is a preferred way of using one's abilities. It is not in itself an ability but rather a preference". Cada individuo posee diferentes estilos de pensamiento y estos a su vez generan diferentes representaciones, es posible detectar por medio de producciones de los individuos a cual estilo de pensamiento se corresponden (Neisser, 2014).

Los estilos de pensamiento influyen en los estilos de pensamiento matemáticos (Borromeo-Ferri, 2004), lo que se traduce en el desarrollo del pensamiento matemático según el estilo preponderante en el individuo. Los estilos de pensamiento matemático (EPM) son atributos de la personalidad, es una preferencia al momento de utilizar una habilidad matemática. Los EPM no son estrategias para la resolución de problemas, ya que estas poseen un gran nivel de afectividad y 
de preferencia (Borromeo-Ferri, 2015), los EPM no son habilidades, sino que más bien son el camino para las habilidades matemáticas tales como la habilidad de argumentar matemáticamente.

Los trabajos de Borromeo-Ferri (2004, 2015), destacan por sistematizar el estudio de los EPM en los niveles escolares, la investigadora ha encontrado tres EPM: formal, visual e integrado. Según Borromeo-Ferri (2004), un individuo analítico o formal es aquel que tiene preferencia por el estilo de pensamiento analítico, se caracteriza por formas internas de imaginación y por representaciones externas formales (se denomina también simbólico), comprende hechos matemáticos preferentemente por medio de la existencia de símbolos o de representaciones verbales, también prefiere proceder en pasos secuenciados (paso a paso).

Un individuo visual es aquel que muestra preferencia por el estilo de pensamiento visual, se caracteriza por imaginar dibujos internos y externalizar representaciones pictóricas, también prefiere para entender hechos y relaciones matemáticas representaciones holísticas, en este caso, las imágenes internas son muchas veces efectos (consecuencia) de asociaciones fuertes con situaciones experimentadas (vividas). El estilo de pensamiento integrado, se puede observar en individuos que combinan el estilo de pensamiento formal con el visual y son bastante flexibles entre las representaciones formales o visuales, el camino que eligen en la solución de los problemas y las formas de proceder con la información.

Además, los estudiantes tienen una Forma de Trabajar el Problema (FTP) que puede ir de lo general a lo particular o viceversa. Como FTP se tiene lo global, local y combinado, donde lo global se refiere a tratar un problema y su solución como un todo; una FTP local se tiene cuando el camino a la solución es tratado paso a paso y una FTP combinado cuando hay en la solución del problema elementos de las dos posibilidades anteriores.

El estudio comparativo realizado por Borromeo-Ferri (2015), muestra que el 53\% de los estudiantes alemanes, junto con el 14\% de estudiantes japoneses y el 29\% de estudiantes sudcoreanos que dan 
respuesta correcta a los problemas, prefieren el EPM formal. Este mismo estudio muestra que la gran mayoría de los estudiantes asiáticos prefieren el EPM integrado. Los EPM permiten dos acercamientos al trabajo con estudiantes, un acercamiento social y otro científico (Borromeo-Ferri, 2014). En lo social se encuentra la mejora en el trabajo con los estudiantes con talento, recomendado ya por Blumen (2008) y por Danielian y Fogarty (2017) que corresponde directamente al trabajo de aula común y donde se hace la diferencia potenciando al estudiante talentoso con diferentes estrategias de acción. Por otro lado, se encuentra el desarrollo científico, como un aporte a la discusión propuesta por Borromeo-Ferri (2014) sobre si los EPM son una preferencia o una capacidad. Además, este estudio determina por primera vez la preferencia de EPM formal, visual e integrado de estudiantes con talento.

En particular, esta investigación se centra en la pregunta: ¿Puede haber alguno de estos tres EPM que sea predominante entre los estudiantes con talento? y si hay alguno predominante, es este EPM determinante al momento de resolver problemas matemáticos. Lo anterior permite abrir una línea no trabajada en relación con el talento matemático, los EPM de los individuos con talento. Así, el objetivo de este trabajo es establecer el estilo de pensamiento matemático predominante que declaran y que luego utilizan los estudiantes con talento académico en la resolución de problemas matemáticos.

Para lograr este objetivo se ha utilizado un cuestionario (BorromeoFerri, 2015) que tiene dos partes, en una parte se pregunta a los estudiantes sobre el estilo que ellos prefieren al momento de resolver problemas (EPM declarado) y en una segunda parte se les enfrenta a la resolución de cuatro problemas matemáticos, para determinar el estilo de pensamiento ejecutado. Para establecer una relación de dependencia de los EPM declarado con las respuestas correctas en la resolución de problemas, se utiliza el test de Ji-cuadrado y se plantean las hipótesis nulas: (1) La obtención de un resultado correcto o incorrecto en el problema es independiente del EPM que declaran los estudiantes y (2) La obtención de un resultado correcto o incorrecto en el problema es independiente de la FTP. 


\section{Método}

Corresponde a un estudio cuantitativo, ex post facto retrospectivo de grupo único (Coolican, 2012). Implica considerar variables atributivas, no manipulables, en contexto natural y con un único grupo de estudiantes. En la tabla 1 se muestran las variables de este estudio junto con sus definiciones.

\section{Tabla 1}

Variables del estudio

\begin{tabular}{|c|c|c|}
\hline Variables & Categoría & Definición \\
\hline \multirow{3}{*}{$\begin{array}{l}\text { Variable1 } \\
\text { Estilos de } \\
\text { Pensamiento } \\
\text { Matemático } \\
\text { (EPM) }\end{array}$} & Formal & $\begin{array}{l}\text { Declara utilizar preferentemente símbolos } \\
\text { y formulas. }\end{array}$ \\
\hline & Visual & $\begin{array}{l}\text { Declara utilizar preferentemente dibujos y } \\
\text { esquematiza la información. }\end{array}$ \\
\hline & Integrado & $\begin{array}{l}\text { Declara utilizar de igual manera símbolos } \\
\text { formales como dibujos o esquemas. }\end{array}$ \\
\hline \multirow{3}{*}{$\begin{array}{l}\text { Variable } 2 \\
\text { Forma de tratar el } \\
\text { problema (FTP) }\end{array}$} & Global & $\begin{array}{l}\text { Declara ver y tratar los problemas } \\
\text { preferentemente de forma general }\end{array}$ \\
\hline & Local & $\begin{array}{l}\text { Declara ver y tratar los problemas } \\
\text { preferentemente desde lo particular. }\end{array}$ \\
\hline & Combinado & $\begin{array}{l}\text { Declara ver y tratar prefiriendo de igual } \\
\text { manera lo particular como lo general. }\end{array}$ \\
\hline \multirow{2}{*}{$\begin{array}{l}\text { Variable } 3 \\
\text { Proceso de } \\
\text { solución aplicado } \\
\text { a la resolución } \\
\text { de problemas } \\
\text { matemáticos } \\
\text { (PSPM) }\end{array}$} & Correcto & \multirow{2}{*}{$\begin{array}{l}\text { En la resolución de problemas el sujeto } \\
\text { activa de manera conjunta el EPM con } \\
\text { la FTP, generando una combinación de } \\
\text { las categorías anteriores para llevar a cabo } \\
\text { un proceso de solución del problema. Este } \\
\text { se ha denominado previamente como } \\
\text { estilo de pensamiento "ejecutado", esto } \\
\text { se observa solo en las producciones del } \\
\text { estudiante en la resolución del problema. }\end{array}$} \\
\hline & Incorrecto & \\
\hline
\end{tabular}




\section{Participantes}

Los sujetos del estudio pertenecen a un programa dirigido a estudiantes con talentos académicos especiales, implementado en una universidad chilena. Este programa favorece el desarrollo de competencias específicas en el área de Matemáticas vinculadas con el proceso de resolución de problemas matemáticos de diversos tipos y mediante estrategias variadas, incluyendo el uso de la tecnología. La aplicación de habilidades de pensamiento lógico para la detección y resolución de problemas, el planteo, modelación e interpretación de problemas contextualizados mediante la Matemática.

La población corresponde a los estudiantes del programa $(\mathrm{N}=260)$, y la muestra de este estudio $(\mathrm{n}=99)$, de carácter intencional voluntaria basada en juicio experto, estuvo compuesta por 46 mujeres y 53 hombres. El promedio de edad estuvo en 14 años y con una desviación de 1.3 años. La composición de la muestra correspondió a 1 estudiante de 5to básico, 1 estudiante de sexto básico, 29 de séptimo básico, 21 de octavo básico, 9 de primero medio y 38 de segundo medio. A todos los estudiantes mayores de 14 años se les pidió su participación voluntaria mediante consentimiento informado. A los estudiantes menores de esa edad, un asentimiento acompañado de una petición de consentimiento a padres.

\section{Medición}

Para la medición y determinación del EPM se aplicó el cuestionario para medir Estilos de Pensamiento Matemático de Borromeo-Ferri, traducción al español y validación realizada en el contexto del proyecto Mathematical Thinking Styles in School and Across Cultures, MaTHSCu-project (Borromeo-Ferri, 2015). El cuestionario traducido al español tiene una validación de contenido por expertos y validado en un estudio piloto arrojando un alfa de Cronbach de .78.

El cuestionario se estructura en dos partes. En la primera parte se mide el EPM declarado con un total de 50 ítems, distribuidos en: 11 ítems referentes al estilo de pensamiento preferido, diez referentes a la 
forma de resolver problemas, nueve referentes al tipo de orientación externa o interna, ocho referentes a la forma de resolver problemas, 11 relacionados con la forma de ver la matemática, seis relacionados con la forma de sentir al momento de aprender matemática, seis relacionados con las condiciones del aprendizaje de la matemática, cuatro relacionados con la forma de ejercitar durante las clases de matemática, tres relacionados con la percepción de satisfacción que se percibe del profesor. Estos ítems se presentan en frases y con respuestas en la escala de Likert (Borromeo-Ferri, 2015): muy de acuerdo, de acuerdo, en desacuerdo, muy en desacuerdo. Segunda parte, se presentan cuatro problemas a resolver con tres ítems referentes al momento de leer el problema, un ítem de desarrollo del problema y cinco ítems referentes a los caminos que favorecieron la solución problema.

La primera parte del instrumento mide las variables EPM y FTP declaradas por los estudiantes. La segunda parte permite observar la variable PSPM, en esta parte el estudiante resuelve 4 problemas. Los 4 ítems que presentan los problemas matemáticos, se evalúan en: respuesta correcta (C) y respuesta incorrecta (I). El análisis de las producciones de los estudiantes, permite categorizar a los estudiantes del programa nuevamente en EPM y FTP de acuerdo a las categorías presentadas en la tabla 1.

\section{Descripción de los problemas}

Problema 1: Ocho personas están en una fiesta de cumpleaños. Cada persona quiere brindar exactamente una vez con los otros asistentes. ¿Cuántas veces sonaran las copas? Este problema es presentado exclusivamente en palabras y de contenido la combinatoria, que se trata normalmente en secundaria.

Problema 2: Carolina tiene una bolsa con 24 bolitas. Ella da la mitad de sus bolitas a Carlos y un tercio de las bolitas a Peter. ¿Con cuántas bolitas se queda Carolina? La situación se plantea en palabras y corresponde a un problema de operatoria básica.

Problema 3: Se presentan cuatro figuras con bloques y se pregunta a) ¿Cuántos bloques habrán en la sexta figura y en la figura número 50 ? 
La situación se plantea de forma gráfica y corresponde a un problema de sucesiones y del termino general del tipo $2 \mathrm{n}+1$.

Problema 4: En el dibujo se ven niños y pizzas. 7 niñas han repartido dos pizzas de manera equitativa y tres niños han repartido una pizza de manera equitativa. ¿Recibe cada niña la misma cantidad de pizza que cada niño?, ¿Quién recibe más y cuánto más? La situación se plantea en palabras y se esquematiza con dibujos, corresponde a un problema de operatoria con fracciones.

Para cada problema se hizo un análisis a priori para describir las respuestas que coinciden con lo visual, con lo analítico y lo que se consideraba integrado. Antes de comenzar a responder al problema y luego de haber leído en detalle, los estudiantes debían indicar cuales habían sido sus primeras impresiones sobre el problema. Luego los estudiantes debían fijar una prioridad en su elección, la cual debía estar relacionada con imágenes, con símbolos o formulas o en los números. Al final del problema se les solicita a los estudiantes responder si se usó un dibujo o un esquema y si el uso de dibujos le había sido de ayuda para comprender el problema y rsponder al problema.

\section{Procedimiento}

En cuanto al procedimiento, los protocolos de implementación del test fueron realizados por los investigadores e implementados con los estudiantes del programa. Los cuestionarios fueron multicopiados a partir de autorización explícita de la autora del cuestionario. Tanto el formato como las instrucciones de aplicación habían sido estrictamente establecidos en el contexto de desarrollo del instrumento y en consecuencia se requirió capacitar a los aplicadores de la prueba. Dos asistentes de investigación fueron entrenados para realizar la aplicación.

La aplicación del cuestionario se realizó en septiembre del 2014, durante las actividades regulares del programa y tuvo una duración de hora y media. La implementación del test se realizó en cuatro visitas de los asistentes al programa y esta transcurrió como establecido, sin preguntas por parte de los sujetos testeados. 
Al termino de cada implementación los ayudantes de investigación recopilaron el material y lo trasladaron para ser tabulados, con lo cual se preparó una base de datos del estudio. La tabulación de datos y corrección de los problemas del instrumento fue ejecutado manualmente por el equipo de trabajo. Luego de esta corrección de los probelmas se procedio al analisis de datos y de la categorización de las producciones escritas, la cual fue realizada por los investigadores responsables del proyecto.

\section{Análisis de datos}

Para el análisis se ejecutó en primer lugar un análisis de supuestos estadísticos, se reemplazaron los datos ausentes y se evaluó la presencia de observaciones atípicas. Con ello se obtuvo una base de datos completa, con valores ausentes imputados, mediante el procedimiento de imputación múltiple (Filzmoser, Garrett \& Reimann, 2005). Además del análisis descriptivo, con los datos se hizo un análisis inferencial aplicando el test de Ji-Cuadrado para establecer la asociación buscada de acuerdo a los objetivos.

En el contraste de las hipótesis con el test Ji - cuadrado, el cual pretende testear la independencia entre grupos, de acuerdo con el criterio de que si el p-valor es menor a .05 se rechaza la hipótesis nula. Principalmente, se pondrá a prueba la independencia entre los grupos formados al inicio de este informe en la sección criterio de clasificación, con los problemas 1, 2, 3. Para llevar a cabo estos contrastes se utilizó el entorno de programación R (R Core Team, 2015), el cual tiene implementado dentro de sus funciones este test.

\section{Resultados}

En primer lugar, se presenta el análisis descriptivo de los datos obtenidos por el cuestionario, donde se divide en la parte de los EPM y las FTP que es declarado por los estudiantes en la primera parte del cuestionario. Luego se presentan los resultados de la segunda parte del 
cuestionario que corresponde a la variable PSMP, donde se analizan las producciones de los estudiantes para el desarrollo de los problemas 1, 2, 3 y 4 . En segundo lugar, se presenta el analisis de independencia entre EPM / FTP y la forma correcta o incorrecta de resolver el problema.

\section{Resultados descriptivos}

Algunos datos de las respuestas de los estudiantes, son de caracter general y permiten tener un contexto del tipo de estudiante que asiste al programa. Uno de estos es el grado de confianza para resolver problemas, donde un $95 \%$ de los estudiantes declara tener una gran confianza en poder resolver problemas y dar respuestas correctas a estos. Otros datos contextuales, son que el $80 \%$ de los estudiantes del programa declara estar interesado en la matemática y que el $93 \%$ de los estudiantes, concuerda en que esta disciplina es aplicable a situaciones reales.

Dentro de las características similares de los estudiantes con talento, se encuentra la persistencia y perseverancia en actividades de la matemática que le motivan y que son de origen metacognitivo, en este caso el 53\% de los estudiantes del programa declara sentirse emocionado y sin temor por el aprendizaje de la matemática.

Según la variable EPM y sus categorías visual, formal o integrado, el instrumento da como resultados el $84 \%$ de los estudiantes del programa prefiere el EPM integrado, que el $8 \%$ es visual y que el $5 \%$ es formal, el resto de los estudiantes aún no tiene una preferencia de EPM.

Para la variable FTP y sus categorías global, local y combinado, el $89 \%$ de los estudiantes del programa prefiere hacer un tratamiento del problema de forma combinada, es decir combinan lo particular (local) con lo general (global) del problema. En cuanto a los procesos de la resolución de problemas matemáticos, un 38\% de los estudiantes declara hacer estos procesos de forma mental, un $25 \%$ prefiere la escritura y un $14 \%$ lo hace de forma integrada, en este caso el resto de los estudiantes no prefiere ninguna de las alternativas presentadas anteriormente.

Con respecto a la categoría PSPM de este estudio, se hace una diferencia entre respuestas correctas (C) e incorrectas (I) de los estudiantes. 
En la tabla 2 se muestra la frecuencia relativa de las respuestas de los estudiantes a los problemas 1, 2, 3 y 4 según EPM y sus categorías formal, visual e integrado. En ella se puede apreciar que el problema 1 y 4 son los que tienen mayor cantidad de respuestas incorrectas y que los problemas 2 y 3 son los que tuvieron mayor cantidad de respuestas correctas.

\section{Tabla 2}

Frecuencia relativa de las respuestas de los estudiantes a los problemas 1, 2, 3 y 4 según EPM declarados en la primera parte del cuestionario.

\begin{tabular}{|c|c|c|c|c|c|c|c|c|c|}
\hline & \multicolumn{2}{|c|}{$\begin{array}{c}\text { Problema } \\
1\end{array}$} & \multicolumn{2}{|c|}{$\begin{array}{c}\text { Problema } \\
2\end{array}$} & \multicolumn{2}{|c|}{$\begin{array}{c}\text { Problema } \\
3\end{array}$} & \multicolumn{2}{|c|}{$\begin{array}{c}\text { Problema } \\
4\end{array}$} \\
\hline & & I & $\mathrm{C}$ & I & $\mathrm{C}$ & I & $\mathrm{C}$ & I & $\mathrm{C}$ \\
\hline \multirow{4}{*}{$\begin{array}{l}\text { Variable } \\
\text { EPM }\end{array}$} & formal & .03 & .02 & .03 & .02 & .04 & .01 & .02 & .03 \\
\hline & visual & .01 & .07 & .07 & .01 & .04 & .04 & .01 & .07 \\
\hline & integrado & .11 & .73 & .52 & .32 & .58 & .26 & .22 & .62 \\
\hline & sin preferencia & .00 & .03 & .00 & .03 & .00 & .03 & .00 & .03 \\
\hline & Total & .15 & .85 & .62 & .38 & .66 & .34 & .25 & .75 \\
\hline
\end{tabular}

Se puede observar en la tabla 2 que para el problema 1 sobre combinatoria, primera columna, que solo un $11 \%$ de los estudiantes que se declaran en la primera parte del cuestionario con un EPM integrado responde de manera adecuada al problea y que un $73 \%$ de este grupo de estudiantes que prefieren este mismo EPM, responde de manera errónea al problema. Algo similar ocurre en el problema 4, donde hay un $22 \%$ de estudiantes que declaran en la primera parte del cuestionario una preferencia por el EPM integrado con respuestas correctas y un $62 \%$ con el mismo EPM pero con respuestas incorrectas.

Para la siguiente categoria FTP sobre la forma de trabajar un problema local, global o combinado, se pueden observar en la tabla 3 las frecuencias relativas de las respuestas de los estudiantes a los problemas 1, 2, 3 y 4 según la variable FTP. 


\section{Tabla 3}

Frecuencia relativa de las respuestas de los estudiantes a los problemas 1, 2, 3 y 4 según FTP declarados en la primera parte del cuestionario

\begin{tabular}{|c|c|c|c|c|c|c|c|c|c|}
\hline & \multicolumn{2}{|c|}{$\begin{array}{c}\text { Problema } \\
1\end{array}$} & \multicolumn{2}{|c|}{$\begin{array}{c}\text { Problema } \\
2\end{array}$} & \multicolumn{2}{|c|}{$\begin{array}{c}\text { Problema } \\
3\end{array}$} & \multicolumn{2}{|c|}{$\begin{array}{c}\text { Problema } \\
4\end{array}$} \\
\hline & & I & $\mathrm{C}$ & I & $\mathrm{C}$ & I & $\mathrm{C}$ & I & $\mathrm{C}$ \\
\hline \multirow{5}{*}{$\begin{array}{l}\text { Variable } \\
\text { FTP }\end{array}$} & Local & .00 & .05 & .01 & .04 & .03 & .02 & .00 & .05 \\
\hline & Global & .01 & .02 & .02 & .01 & .02 & .01 & .01 & .02 \\
\hline & Combinado & .14 & .75 & .59 & .30 & .60 & .29 & .24 & .65 \\
\hline & sin preferencia & .00 & .03 & .00 & .03 & .01 & .02 & .00 & .03 \\
\hline & Total & .15 & .85 & .62 & .38 & .66 & .34 & .25 & .75 \\
\hline
\end{tabular}

En la figura 1 se puede observar que el problema 1 fue resuelto correctamente por un $15 \%$ de los estudiantes del programa, de estos estudiantes, gran parte declaro como preferencia el EPM integrado y el $84 \%$ de los estudiantes no responde de forma correcta este problema. Algo similar ocurre en el problema 4, donde solo el 25\% responde correctamente al problema y la gran mayoría corresponde a EPM integrado. En cambio, el 62\% de los estudiantes del programa respondió correctamente el problema 2, de estos estudiantes gran parte fue clasificado en la categoría EPM integrado. También el 66\% de los estudiantes del programa respondió correctamente la pregunta 3, donde $58 \%$ fue clasificado en la categoría EPM integrado declarado.

La figura 1 muestra la comparación entre los resultados correctos según los problemas. Se puede ver que los problemas 1 y 4 no tuvieron el mayor porcentaje de resultados correctos y los problemas 2 y 3 si tienen mayor porcentaje de respuestas correctas versus respuestas incorrectas. Aquí el problema 1 de combinatoria presenta un grado mayor de dificultad a los estudiantes participantes del programa. En cambio el problema 3 de sucesiones y presentado inicialmente con dibujos de bloques, es respondido de manera correcta. 


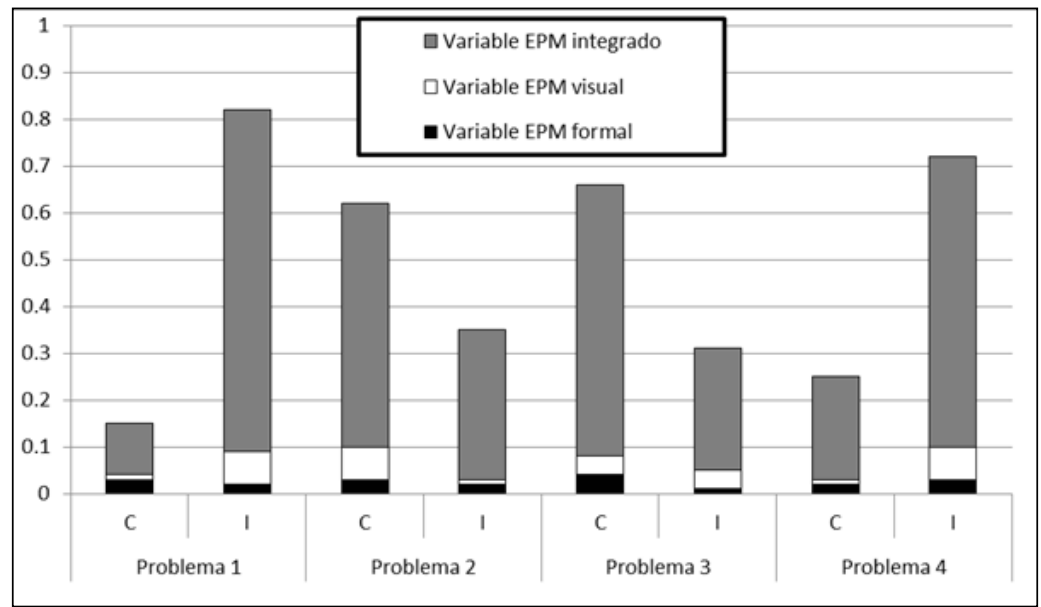

Figura 1. Respuestas correctas (C) e incorrectas (I) de los estudiantes a los problemas 1, 2, 3 y 4 según los EPM declarados.

En el problema 3, se puede apreciar que la gran mayoría de los estudiantes integrados prefiere imaginarse la situación y buscar una fórmula. Para los problemas 1, 2 y 4 hay una mayor coincidencia entre la preferencia integrada y la forma de resolver los problemas.

El análisis de las producciones de los estudiantes frente al desarrollo de los problemas $1,2,3$ y 4 , presenta 10 posibilidades, relacionadas con la combinación de los diferentes tipos de las dos categorías: formalglobal, formal-local, formal-combinado, visual-global, visual-local, visual-combinado, integrado-global, integrado-local, integrado-combinado y sin desarrollo que corresponde a los estudiantes que no hicieron un desarrollo. En la tabla 4 se muestran los resultados de los estudiantes según las 9 categorías para la variable PSPM y si la respuesta es correcta o incorrecta. 


\section{Tabla 4}

Frecuencia relativa de estudiantes del programa de acuerdo a la variable PSPM y las respuestas correctas e incorrectas a los problemas 1, 2, 3 y 4

Problema 1 Problema 2 Problema 3 Problema 4

\begin{tabular}{lcccccccc} 
Variable PSPM & $\mathrm{C}$ & $\mathrm{I}$ & $\mathrm{C}$ & $\mathrm{I}$ & $\mathrm{C}$ & $\mathrm{I}$ & $\mathrm{C}$ & $\mathrm{I}$ \\
\hline Formal-global & .01 & .36 & .24 & .12 & .03 & .02 & .14 & .11 \\
Formal-local & .00 & .05 & .24 & .12 & .56 & .12 & .01 & .04 \\
Formal-combinado & .00 & .00 & .00 & .00 & .00 & .00 & .00 & .00 \\
Visual-global & .03 & .06 & .00 & .01 & .00 & .00 & .00 & .00 \\
Visual-Local & .04 & .02 & .01 & .01 & .05 & .03 & .05 & .26 \\
Visual-combinado & .00 & .00 & .00 & .00 & .00 & .00 & .00 & .00 \\
Integrado-global & .01 & .03 & .00 & .00 & .00 & .00 & .00 & .00 \\
Integrado-local & .02 & .01 & .00 & .00 & 00 & .00 & .00 & .00 \\
Integrado-combinado & .04 & .19 & .07 & .02 & .00 & .00 & .00 & .00 \\
Sin desarrollo & .00 & .12 & .05 & .10 & .02 & .17 & .05 & .33 \\
\hline \multicolumn{1}{c}{ Total } & .15 & .85 & .62 & .38 & .66 & .34 & .25 & .75
\end{tabular}

En la tabla 4, se puede apreciar lo formal en las tres primeras filas de resultados, esto nos indica que la mayoría de los estudiantes del programa ejecutan el estilo de pensamiento formal. En el problema $1 \mathrm{el}$ $42 \%$ trabaja con lo formal independiente de si su resultado es correcto o incorrecto, en el problema 2 el $72 \%$ trabaja con lo formal, en el problema 3 el $73 \%$ trabaja con lo formal y en el problema 4, solo el $30 \%$ trabaja con lo formal. Esto significa que la mayoría de los estudiantes tiene un PSPM (ejecutado) formal, es decir, los resultados de respuestas abiertas, hay una preferencia ejecutada que prefiere lo formal.

El 15\% de los estudiantes del programa respondió correctamente el problema 1 y gran parte de los estudiantes que procedieron de forma integrada, tuvo el problema incorrecto, por otro lado el $36 \%$ de los estudiantes resuelve el problema utilizando una forma más bien analítica. En el problema 2, 3 y 4, nuevamente se ve una preferencia por las 
expresiones formales y dada la estructura del problema no se observó una diferencia entre lo global y lo local. Por otro lado, el 19\% de la muestra que se declara integrado resuelve el problema 1 de manera integrada, aunque con solución incorrecta.

El problema 1 relativo a combinatoria y el problema 2 relativo a operatoria básica, no fueron presentados con dibujos. Frente a la pregunta si les ayuda un dibujo para responder el problema el 54\% de los estudiantes que responden de forma incorrecta al problema 1 indican que si les ayudó un dibujo en la respuesta. En el caso del problema 2, un $66 \%$ indica que si les ayuda un dibujo, este porcentaje se desglosa en un $32 \%$ de resultados correctos y en un $23 \%$ de resultados incorrectos. Algo similar al problema 1 , ocurre en el problema 4 de fracciones, donde también hay un $66 \%$ de estudiantes que indican que si les ayuda un dibujo pero donde un $42 \%$ de los estudiantes responden de manera incorrecta al problema. En el caso del problema 3, un 55\% indica que les ayuda un dibujo para resolver el problema, donde el $37 \%$ de estos estudiantes resuelven de manera correcta. Aunque todos los estudiantes indican que un dibujo les podría ayudar a resolver un problema, muy pocos de ellos utilizan un dibujo de manera adecuada un dibujo para resolver los problemas, en el problema 1 , solo un $3 \%$, en el problema 2 solo $14 \%$, en el problema 3, un $19 \%$ y en el problema 4 un $6 \%$.

Los estudiantes que tuvieron correcta la solución al problema 1 , se ayudaron de un dibujo, lo cual corresponde a un EPM visual y en la misma cantidad de estudiantes que respondieron adecuadamente no utilizaron un dibujo. En el caso del problema 2, donde hubo una mayor cantidad de respuestas correctas, se puede apreciar que el uso del dibujo no juega un rol importante para la solución del problema, ya que la preferencia de 76 que se obtiene de las preferencias "sin dibujo" y "no utiliza dibujo" indica que la utilización de un dibujo no es suficiente para resolver el problema. Lo mismo ocurre en el problema 3, donde la no utilización de un dibujo no es suficiente al momento del desarrollo del problema. En el problema 4, ocurre el mismo fenómeno que en el problema 1, donde 14 estudiantes declaran que el dibujo les ayudó y 19 no utilizaron dibujos para resolverlo. 


\section{Resultados prueba de independencia}

En la siguiente sección se presenta el análisis inferencial, en el que se ponen a prueba las 2 hipótesis nulas:

(1) H1_X La obtención de un resultado correcto o incorrecto en el problema $\mathrm{X}$ es independiente del estilo de pensamiento matemático (EPM, visual, formal, integrado) que declaran los estudiantes.

(2) H2_X La obtención de un resultado correcto o incorrecto en el problema $\mathrm{X}$ es independiente de la forma del tratamiento que se le da al problema (FTP, global, local, combinado)

Ambas hipótesis nos permiten hablar de la dependencia o independencia de los estilos de pensamiento matemático declarados con los ejecutados (PSPM). En lo que sigue $\mathrm{X}$ es reemplazado por el número del problema de desarrollo 1, 2, 3 o 4, que fueron presentados en la sección de método.

En el análisis de la prueba de independencia del problema 1 sobre combinatoria, se obtuvo $p=.035$, por lo que se rechaza la hipótesis nula. Entonces, la obtención de un resultado correcto en el problema 1 es dependiente del EPM, visual, formal, integrado que declaran los estudiantes en la primera parte del cuestionario. El 84\% de los estudiantes declaran en la primera parte del cuestionario que prefieren el EPM integrado, por otro lado el $85 \%$ responde manera incorrecta a este problema y trabaja con los EPM formal (41\%) e integrado (23\%), lo cual nos indica para la gran mayoría de los estudiantes el EPM es determinante al momento de resolver un problema de combinatoria y que habría una contradicción entre algo que estarían aprendiendo y su propio EPM. Las producciones de los estudiantes que responden de manera correcta a este ítem pertenecen al grupo de visual-local con un $4 \%$ e integrado-combinado con un $4 \%$.

En el caso de la hipótesis $\mathrm{H}_{2} \_1$ se obtuvo $p=.52$, con lo cual no se rechaza la hipótesis y se puede decir que las respuestas correcta o incorrecta es independiente de la categoría FTP (global, local o combinado). El $89 \%$ de los estudiantes declaran en la primera parte del cuestionario una preferencia hacia la FTP combinado de acercarse a un 
problema, de este grupo el 14\% responde de manera correcta (ver tabla 3) pero solo el $4 \%$ de este grupo utiliza esta FTP en sus producciones (segunda parte del cuestionario).

En el análisis de la prueba de independencia del problema 2 sobre operatoria básica se obtuvo $p=.069$ con lo cual no se rechaza esta hipótesis y se puede decir que la obtención de un resultado correcto para el problema 2 de operatoria básica es independiente de la categoría EPM (visual, formal o integrado). Dado que el $62 \%$ de los estudiantes responden de forma correcta al problema y el $48 \%$ de estas respuestas corresponden a los grupos formal-global (24\%) y formal-local (24\%), es independiente lo declarado sobre la preferencia del estilo integrado (primera parte del cuestionario 84\% EPM integrado) y lo ejecutado en la solución del problema solamente formal (segunda parte del cuestionario).

En el caso de la hipótesis H2_2, se obtuvo $p=.027$, con lo cual se puede decir que los resultados correctos o incorrectos para el problema 2 de operatoria básica es dependiente de la categoría FTP (global, local o combinado). En este caso hay un $7 \%$ de respuestas correctas que están en el grupo de integrado-combinado y el $48 \%$ corresponden a global o formal.

En el análisis de la prueba de independencia del problema 3 sobre sucesiones, al poner a prueba H1_3 y H2_3 se obtuvo $p$ levemente mayores que .05 ( $p=.063$ para H1_3 y $p=.674$ para H2_3) con lo cual no se rechazan estas hipótesis. Con lo cual se puede decir que las respuestas son independientes del EPM y de FTP. En este caso, el 66\% de los estudiantes responden de forma correcta al problema 3 y el $56 \%$ de estas respuestas correctas corresponde al grupo formal-local. Esto, en relación con los otros dos problemas anteriores nos indica que los estudiantes prefieren un EPM integrado pero que al momento de resolver un problema, estarían utilizando en su mayoría el EPM formal.

En el caso de FTP hay un 61\% de producciones de estudiantes que corresponden a la forma de trabajo local y lo declarado en la primera parte del cuestionario con respecto a la FTP fue de un $89 \%$ de preferencia por lo combinado. 
En el análisis de la prueba de independencia del problema 4 sobre fracciones, para la hipótesis nula $\mathrm{H} 1 \_4$ se obtuvo $p=.503$, con lo cual no se rechaza la hipótesis y las respuestas dadas por los estudiantes en la segunda parte del cuestionario son independientes del EPM declarado en la primera parte del cuestionario. En este caso, el 25\% de los estudiantes responden de forma correcta al problema 4 y de este el 15\% corresponde a un EPM formal.

Según los resultados del análisis de los problemas 2, 3 y 4 y para la categoría EPM se puede decir que la obtención de un resultado correcto o incorrecto es independiente del EPM visual, formal o integrado que declaran los estudiantes en la primera parte del cuestionario. Los estudiantes declaran en un 84\% un EPM integrado y al momento de resolver un problema trabajan de preferencia con el EPM formal (ver tabla 4).

Para la hipótesis H2_4, se obtuvo $p=.392$, con lo cual no se rechaza esta hipótesis y se puede decir que la obtención de un resultado correcto es independiente de la categoría FTP global, local o combinado. El problema 4 sobre fracciones fue resuelto de manera correcta por el $25 \%$ de los estudiantes del programa y el 14\% de las respuestas correctas utiliza una FTP global. La misma independencia de la FTP utilizada se presenta en los problemas 1, 3 y 4, donde el problema 3 sobre sucesiones presenta el mayor logro de respuestas correctas $66 \%$.

Así, los resultados de la prueba de independencia entre las variables del estudio muestran que para el problema 1 hay una dependencia del EPM declarado y el utilizado en la solución del problema. En el problema 2, 3 y 4 hay una independencia del EPM declarado y las respuestas correctas o incorrectas a los problemas. Por último, en el problema 1, 3 y 4 hay una independencia de la FTP y las respuestas correctas o incorrectas.

\section{Discusión}

Uno de los resultados de esta investigación muestra que los jóvenes con talento académico declaran una preferencia por el EPM integrado 
y una FTP combinado, lo cual da un indicio sobre las necesidades académicas de estos estudiantes y reformular nuevas estrategias de instrucción (Blumen, 2008) donde el EPM integrado juegue un rol en los programas especializados en talento académico y donde se fortalezca la FTP combinada. Concordamos en este sentido con Zhang (2017) que promueve y fortalece la noción de estilo intelectual propio, notando las diferencias que esta investigación hace en el Estilo de Pensamiento Matemático de jóvenes con talento.

En el estudio presentado por Borromeo-Ferri (2015), se indica que la preferencia de los EPM depende también de otros factores, sorprende que los estudiantes con talento prefieran el mismo EPM que los estudiantes asiáticos que tienen resultados correctos en el mismo cuestionario. Por otro lado, es sorprendente que los estudiantes con talento participantes de este estudio y del programa, respondan de manera adecuada a solo dos de los cuatro problemas presentados. Este resultado podría deberse a que hay una contradicción entre el EPM preferido y el utilizado en la resolución de problemas o bien según a lo propuesto por Reyes y Karg (2009) no se cumpliría con una de las características de estudiante con talento matemático como lo es el dominio de campos del conocimiento matemático.

Una de las limitaciones de este estudio es la ausencia de un grupo comparativo, por eso se presenta para comparar los resultados de jóvenes no chilenos que están en el noveno o décimo año escolar del estudio realizado por Borromeo-Ferri (2015). Aunque nuevamente esta comparación tiene sus limitaciones, ya que no es posible comparar estudiantes con talento académico con otros grupos que no sean similares. Una posibilidad para superar esta limitante, sería hacer el mismo estudio considerando dos grupos de estudiantes con talento, donde uno de los grupos tenga los mismos intereses académicos o bien separar de manera aleatoria a un grupo grande de estudiantes con talento o bien considerar estudiantes con talento de la misma edad y de otros países similares a Chile.

Los estudiantes del programa utilizan en la resolución de problemas un EPM formal que es lo contrapuesto a lo intuitivo. Según Sak 
(2004) los estudiantes con talento prefieren la intuición al momento de resolver problemas, la intuición por otra parte estaría más relacionada con lo visual, lo cual también comparte Martínez y Guirado (2012) y muy pocos de los estudiantes del programa utilizan en sus producciones el EPM visual. En este caso, se podría establecer que la contradicción que presentan los estudiantes del estudio entre el EPM declarado y el EPM ejecutado no les permite resolver de manera adecuada a dos de los cuatro problemas. Esta contradicción podría deberse a que el sistema escolar tiende a enseñar y privilegiar un acercamiento a la matemática más formal y por ende la escuela desarrolla un EPM formal, sin desarrollar el EPM preferente por el individuo o bien a que los profesores de matemática tienden a tener un EPM formal y este es el que predomina en clases.

Concordamos con lo que promueve Blumen (2008) sobre el trabajo con niños con talento y con lo recién propuesto por Danielian y Fogarty (2017) en ambos casos, la postura es promover desde las diferencias de los estudiantes, reconociendo que hay diferentes formas y estilos. Los nińos con talento necesitan más que un ambiente estimulante, en el caso de la matemática, agregamos que la sala de clases no es suficiente para desarrollar talento matemático. Aunque todos los jóvenes deben ser motivados y a todos se les debe dar lo que requieren para el logro del aprendizaje, reconocemos la necesidad de potenciar a los estudiantes con talento y potenciarlos en el interés por la matemática, especialmente se debe recurrir a los rasgos propios de la personalidad como los EPM en programas especializados de talentos. Un futuro estudio podría estar basado en la pregunta ¿Qué tipo de EPM debería tener el docente de un programa de talento académico para potenciar al estudiante con talento?

Un alto porcentaje de los estudiantes declara sentirse confiado y motivado frente a la resolución de determinados problemas matemáticos, como también la mayoría de los estudiantes afirma sentirse emocionado al aprender matemáticas, esto nos indica que los estudiantes tienen un alto grado de confianza en sí mismo, lo cual es comentado recientemente en Gómez-Arizaga y otros (2016), lo que les 
permite resolver de buena manera 2 de los cuatro problemas presentados. Con respecto a esta situación de sobre confianza que presentan los estudiantes del programa, entendido como un optimismo exagerado en la resolución de problemas matemáticos (Macbeth, 2009), se debe determinar las condiciones por la cual ocurre este fenómeno y especificar si es un propósito del programa el fortalecer esta confianza.

Aunque la gran mayoría de los estudiantes de la muestra declaran como preferencia el EPM integrado, solo un 19\% desarrolla de acuerdo a este estilo en el problema 1 . Por otro lado, aunque se declaran con EPM integrado, solo el $56 \%$ de la muestra resuelve el problema 3 de manera formal con resultado correcto, esto se debe a que el problema ya presenta información icónica explicitada en su enunciado. Cabe destacar, que las diferencias significativas entre el uso de un dibujo y las respuestas correctas y el no uso de un dibujo y las respuestas incorrectas, nos evidencia que el estilo que permite llegar a un resultado correcto, debería ser el EPM integrado.

\section{Referencias}

Aretzaga, L. (Ed.). (2013). Orientaciones educativas. Alumnado con altas capacidades intelectuales. Vitoria-Gasteiz: Eusko Jaurlaritzaren Argitalpen Zerbitzu Nagusia.

Artola T., Barraca, J. \& Mosteiro, P. (2005). Niños con altas capacidades: quiénes son y cómo tratarlos. Madrid: Entha.

Blumen, S. (2008). Motivación, sobredotación y talento: un desafío para el éxito. Revista de Psicología, 26(1), 147-184.

Borromeo-Ferri, R. (2004). Mathematische Denkstile. Ergebnisse einer empirischen Studie. Hildesheim: Franzbecker.

Borromeo-Ferri, R. (2014). Präferenzen oder Fahigkeiten? - Mathematische Denkstile im Span- nungsfeld von Persönlichkeit, Kultur und schulischer Sozialisation. Beiträge zum Mathematikunterricht, 13-20. 
Borromeo Ferri, R. (2015). Zur Rolle kognitiver Aspekte in der Modellierungsdiskussion. En G. Kaiser y H. W. Henn (Eds.), Werner Blum und seine Beiträge zum Modellieren im Mathematikunterricht. Wiesbaden: Springer, 63-76. https://doi. org/10.1007/978-3-658-09532-1_5

Bransford, J. D., Brown, A. L. \& Cocking, R. (Eds.). (2002). How People Learn. Brain, Mind, Experience, and School. Washington. DC: Committee on Developments in the Science of Learning.

Coolican, H. (2012). Métodos de investigación y estadística en psicología, 3ra edición traducción de la 4ta edición en inglés. México: El Manual Moderno.

Danielian, J. \& Fogarty, E. (2017). Teaching Gifted Children: Success Strategies for Teaching High-Ability Learners. USA: National Association for Gifted Chiledren.

Filzmoser, P., Garrett, R. G. \& Reimann, C. (2005). Multivariate outlier detection in exploration geochemistry. Computers \& Geosciences, 31, 579-587. https://doi.org/10.1016/j.cageo.2004.11.013

Gómez-Arizaga, M. P., Conejeros-Solar, M., Sandoval-Rodríguez, K. \& Armijo-Solís, S. (2016). Doble excepcionalidad: análisis exploratorio de experiencias y autoimagen en estudiantes chilenos. Revista de Psicología, 34(1), http://dx.doi.org/10.18800/ psico.201601.001

Martínez, M. y Guirado, A. (2012). Altas capacidades intelectuales. Pautas de actuación, orientación, intervención y evaluación en el periodo escolar. Barcelona: Graó.

Macbeth, G. (2009). Distorsiones del éxito subjetivo en la resolución de problemas matemáticos. Boletín de Psicología, 95(1), 59-72.

Montero, I. y León, O. (2002). Métodos de investigación en psicología y educación. Madrid: McGraw-Hill.

Neisser, U. (2014) Cognitive Psychology: Classic Edition. New York: Psychology Press.

R. Core Team. (2013). R: A language and environment for statistical computing. $R$ Foundation for Statistical Computing. Viena: $\mathrm{R}$ Core Team. 
Rankin, J. G. (2017). Engaging and Challenging Gifted Students: Tips for Supporting Extraordinary. USA: ASCD.

Reyes, P. y Karg, A. (2009). Una aproximación al trabajo con niños especialmente dotados en matemáticas. En M. J González, M. T. González y J. Murillo (Eds.), Investigación en Educación Matemática XIII. Santander: Sociedad Española de Investigación en Educación Matemática, SEIEM, 403-414.

Sak, U. (2004). A Synthesis of Research on Psychological Types of Gifted Adolescents. The Journal of Secondary Gifted Education, $X V(2), 70-79$. https://doi.org/10.4219/jsge-2004-449

Sriraman, B. (2005). Are Giftedness and Creativity Synonyms in Mathematics? The Journal of Secondary Gifted Education, $X V I I(1), 20-36$.

Sternberg, R. (1994). Allowing for thinking styles. Educational Leadership, 52(3), 36-40.

Sternberg, R. (1997). Thinking Styles. New York: Cambridge University Press. https://doi.org/10.1017/CBO9780511584152

Sternberg, R. J. (2007). Wisdom, Intelligence, and Creativity Synthesized. New York: Cambridge University Press.

Tarazona, J. (2011). Experiencia en la atención a alumnos con talento y superdotación en el ámbito escolar. Consensus, 16(1), 91-97.

Zhang, L. (2017). The Value of Intellectual Styles. New York: Cambridge University Press. https://doi.org/10.1017/9781316014561

Recibido: 13 de mayo, 2015

Revisado: 22 de agosto, 2017 Aceptado: 29 de septiembre, 2017 